PROCEEDINGS OF THE

AMERICAN MATHEMATICAL SOCIETY

Volume 131, Number 8, Pages 2379-2383

S 0002-9939(03)07053-9

Article electronically published on March 18, 2003

\title{
MAXIMAL MONOTONICITY, CONJUGATION AND THE DUALITY PRODUCT
}

\author{
REGINA SANDRA BURACHIK AND B. F. SVAITER
}

(Communicated by Jonathan M. Borwein)

\begin{abstract}
Recently, the authors studied the connection between each maximal monotone operator $T$ and a family $\mathcal{H}(T)$ of convex functions. Each member of this family characterizes the operator and satisfies two particular inequalities.

The aim of this paper is to establish the converse of the latter fact. Namely, that every convex function satisfying those two particular inequalities is associated to a unique maximal monotone operator.
\end{abstract}

\section{INTRODUCTION}

Let $X$ be a real Banach space and $X^{*}$ be the dual of $X$. Denote by $\langle\cdot, \cdot\rangle$ the duality product. A multivalued operator $T: X \rightrightarrows X^{*}$ is monotone if

$$
\left\langle x_{1}-x_{2}, v_{1}-v_{2}\right\rangle \geq 0, \quad \forall v_{1} \in T\left(x_{1}\right), v_{2} \in T\left(x_{2}\right) .
$$

Such an operator is maximal monotone if its graph, that is, the set

$$
G(T)=\left\{(x, v) \in X \times X^{*} \mid v \in T(x)\right\},
$$

is not properly contained in the graph of any other monotone operator $T^{\prime}: X \rightrightarrows X^{*}$. We will identify $T$ with its graph $G(T)$.

The subdifferential of a function $f: X \rightarrow \overline{\mathbb{R}}$ is the multivalued operator $\partial f$ : $X \rightrightarrows X^{*}$ defined by

$$
\partial f(x)=\left\{v \in X^{*} \mid f(y) \geq f(x)+\langle y-x, v\rangle, \forall y \in X\right\} .
$$

A convex function is closed if it is lower semicontinuous and it is proper if it does not attain the value $-\infty$, and is not $+\infty$ everywhere.

Rockafellar [7] proved that subdifferentials of proper closed convex functions on $X$ are maximal monotone. In general, maximal monotone operators are not subdifferentials of convex functions. Krauss [5] managed to represent maximal monotone operators by subdifferentials of saddle functions on $X \times X$. After that, Fitzpatrick 4 proved that maximal monotone operators can be represented by convex functions on $X \times X^{*}$. This result has been recently rediscovered in [6, 2].

Received by the editors February 28, 2002.

2000 Mathematics Subject Classification. Primary 47H05.

Key words and phrases. Convex functions, maximal monotone operators, duality product, conjugation.

The first author was partially supported by CNPq and by PRONEX-Optimization.

The second author was partially supported by CNPq Grant 301200/93-9(RN) and by PRONEX-Optimization. 
Next we describe Fitzpatrick's results. Given a maximal monotone operator $T: X \rightrightarrows X^{*}$, define

$$
\mathcal{H}(T):=\left\{\begin{array}{l|l}
h: X \times X^{*} \rightarrow \overline{\mathbb{R}} & \begin{array}{l}
h \text { convex, closed; } \\
\forall(x, v) \in X \times X^{*}, h(x, v) \geq\langle x, v\rangle, \\
(x, v) \in T \Rightarrow h(x, v)=\langle x, v\rangle .
\end{array}
\end{array}\right\} .
$$

Also define $\varphi_{T}: X \times X^{*} \rightarrow \overline{\mathbb{R}}$

$$
\varphi_{T}(x, v):=\sup \{\langle x-y, u-v\rangle \mid(y, u) \in T\}+\langle x, v\rangle .
$$

Theorem 1.1 (Fitzpatrick [4]). Let $T: X \rightrightarrows X^{*}$ be maximal monotone. The function $\varphi_{T}$ belongs to $\mathcal{H}(T)$ and is the smallest function of this family. Moreover, for any $h \in \mathcal{H}(T)$,

$$
(x, v) \in T \Leftrightarrow h(x, v)=\langle x, v\rangle .
$$

From the above equivalence, it follows that each $h \in \mathcal{H}(T)$ fully characterizes $T$.

Given a function $f: X \rightarrow \overline{\mathbb{R}}$, the Legendre transform or conjugate of $f$ is defined as $f^{*}: X^{*} \rightarrow \overline{\mathbb{R}}$

$$
f^{*}(v)=\sup \{\langle x, v\rangle-f(x) \mid x \in X\} .
$$

Conjugation is an essential tool in the study of convex functions. Let $f$ be a proper convex function. From the previous definitions, we have the Fenchel-Young inequality: for all $x \in X, v \in X^{*}$,

$$
f(x)+f^{*}(v) \geq\langle x, v\rangle, f(x)+f^{*}(v)=\langle x, v\rangle \Longleftrightarrow v \in \partial f(x) .
$$

For $h: X \times X^{*} \rightarrow \overline{\mathbb{R}}$, the conjugate of $h$ is defined on $X^{*} \times X^{* *}$. Since there is a natural injection of $X$ into $X^{* *}$, we define $\mathcal{J}(h): X \times X^{*} \rightarrow \overline{\mathbb{R}}$,

$$
\begin{aligned}
\mathcal{J}(h)(x, v) & =h^{*}(v, x) \\
& =\sup \left\{\langle(y, u),(v, x)\rangle-h(y, u) ; \mid(y, u) \in X \times X^{*}\right\} \\
& =\sup \left\{\langle y, v\rangle+\langle x, u\rangle-h(y, u) ; \mid(y, u) \in X \times X^{*}\right\} .
\end{aligned}
$$

Fitzpatrick [4. Prop. 4.2] proved that if $T: X \rightrightarrows X^{*}$ is maximal monotone, $\mathcal{J}\left(\varphi_{T}\right)$ also belongs to $\mathcal{H}(T)$. In [2 this result was extended to any $h \in \mathcal{H}(T)$. Namely, if $h \in \mathcal{H}(T)$, then $\mathcal{J}(h) \in \mathcal{H}(T)$. Altogether, the result in [2] can be expressed as the implication

$$
\begin{array}{ll}
T: X \rightrightarrows X^{*} \text { maximal monotone } & \Rightarrow \begin{array}{l}
\forall(x, v) \in X \times X^{*} \\
h \in \mathcal{H}(T)
\end{array} \\
& h(x, v) \geq\langle x, v\rangle, h^{*}(v, x) \geq\langle x, v\rangle .
\end{array}
$$

Our aim is to prove the converse of this implication in a reflexive Banach space. Namely,

$$
\begin{aligned}
& h: X \times X^{*} \rightarrow \overline{\mathbb{R}} \text { convex, lsc, } \\
& \forall(x, v) \in X \times X^{*}, \\
& h(x, v) \geq\langle x, v\rangle, h^{*}(v, x) \geq\langle x, v\rangle .
\end{aligned} \quad \Rightarrow \begin{aligned}
& \exists ! T: X \rightrightarrows X^{*} \text { maximal monotone } \\
& h \in \mathcal{H}(T)
\end{aligned}
$$

The paper is organized as follows. In Section 2 we state some necessary previous results. The last section contains the formal statement and the proof of the implication above (see Section 3. Theorem 3.1). 


\section{TheORETICAL PRELIMINARIES}

We include in this section theoretical results which are necessary for the proof of Theorem 3.1 . From now on $X$ is a real Banach space.

Theorem 2.1 ([2] Theor. 5.3]). Let $T: X \rightrightarrows X^{*}$ be maximal monotone. Then, the operator $\mathcal{J}$ maps $\mathcal{H}(T)$ into itself.

We also assume from now on that $X$ is reflexive. Asplund [1] has shown that, in this case, there exists an equivalent norm on $X$ which is everywhere Gâteaux differentiable except at the origin and whose polar norm on $X^{*}$ is everywhere Gâteaux differentiable except at the origin. For simplifying the notation, we assume that the given norm on $X$ already has these special properties. We use the same notation $\|\cdot\|$ for this norm on $X$ and its associated norm on the dual $X^{*}$. Denote by $J$ the Gâteaux gradient of the function $g(x)=(1 / 2)\|x\|^{2}$. Thus, $J$ is the duality mapping, which assigns to each $x \in X$ the unique $J(x) \in X^{*}$ such that

$$
\langle x, J(x)\rangle=\|x\|^{2}=\|J(x)\|^{2} .
$$

The inverse of this duality mapping will be denoted by $J_{*}$, which is the subgradient of the function $g^{*}(v)=(1 / 2)\|v\|^{2}$.

The following result was proved in [8, Section 2], where it appears as a corollary.

Proposition 2.2. Let $T: X \rightrightarrows X^{*}$ be a monotone operator. Under the above assumptions, in order for $T$ to be maximal monotone, it is necessary and sufficient that $(T+J): X \rightrightarrows X^{*}$ be onto.

Using the fact that $\langle z, u\rangle \geq-\|z\|\|u\|$ for all $z \in X, u \in X^{*}$, one can easily obtain the proposition below.

Proposition 2.3. Under the above assumptions, let $z \in X, u \in X^{*}$. Then

$$
\|z\|^{2}+\|u\|^{2}+2\langle z, u\rangle \geq 0,
$$

with equality if and only if $u=-J(z)$ (or equivalently $z=-J_{*}(u)$ ).

\section{MAin RESUlT}

Now we state formally and prove the main result. Recall that $X$ is a reflexive real Banach space. For convenience, $X$ has been Asplund-renormed.

Theorem 3.1. Under the above assumptions, let $h: X \times X^{*} \rightarrow \overline{\mathbb{R}}$ be a convex lower semicontinuous function. Suppose that

$$
\forall(x, v) \in X \times X^{*}, \quad h(x, v) \geq\langle x, v\rangle, \quad h^{*}(v, x) \geq\langle x, v\rangle .
$$

Define

$$
T=\left\{(x, v) \in X \times X^{*} \mid h(x, v)=\langle x, v\rangle\right\} .
$$

Then $T$ is maximal monotone and $h, \mathcal{J}(h) \in \mathcal{H}(T)$.

Proof. First we claim that $T$ is monotone. Indeed, take $v_{1} \in T\left(x_{1}\right), v_{2} \in T\left(x_{2}\right)$; then

$$
\left\langle x_{1}, v_{1}\right\rangle=h\left(x_{1}, v_{1}\right), \quad\left\langle x_{2}, v_{2}\right\rangle=h\left(x_{2}, v_{2}\right) .
$$

The convexity of $h$ together with (3.1) gives

$$
\begin{aligned}
(1 / 2)\left(h\left(x_{1}, v_{1}\right)+h\left(x_{2}, v_{2}\right)\right) & \geq h\left((1 / 2)\left(x_{1}+x_{2}\right),(1 / 2)\left(v_{1}+v_{2}\right)\right) \\
& \geq\left\langle(1 / 2)\left(x_{1}+x_{2}\right),(1 / 2)\left(v_{1}+v_{2}\right)\right\rangle .
\end{aligned}
$$


Combining this with (3.3) we obtain

$$
(1 / 2)\left(\left\langle x_{1}, v_{1}\right\rangle+\left\langle x_{2}, v_{2}\right\rangle\right) \geq(1 / 4)\left\langle x_{1}+x_{2}, v_{1}+v_{2}\right\rangle,
$$

which is equivalent to $\left\langle x_{1}-x_{2}, v_{1}-v_{2}\right\rangle \geq 0$.

Now we claim that $T+J: X \rightrightarrows X^{*}$ is onto. To prove this fact, take an arbitrary $v_{0} \in X^{*}$ and define $\varphi: X \times X^{*} \rightarrow \mathbb{R} \cup\{+\infty\}$,

$$
\begin{aligned}
\varphi(x, v) & :=(1 / 2)\left(\|x\|^{2}+\left\|v-v_{0}\right\|^{2}+2\left\langle x, v-v_{0}\right\rangle\right)+(h(x, v)-\langle x, v\rangle) \\
& =(1 / 2)\left(\left\|v-v_{0}\right\|^{2}+\|x\|^{2}\right)-\left\langle v_{0}, x\right\rangle+h(x, v),
\end{aligned}
$$

where $\|\cdot\|, J$ are the norm and duality map defined above, respectively. By the first expression for $\varphi$, assumptions (3.1)-(3.2) and Proposition 2.3 we have $\varphi \geq 0$, with equality only if $v-v_{0}=-J(x)$ and $v \in T(x)$. This implies $v_{0} \in(T+J)(x)$. The second expression of $\varphi$ shows that this function is the sum of a differentiable convex function plus a lower semicontinuous convex function. By [9, Th. 3] or [3. p. 62] the subdifferential of this sum is the sum of the subdifferentials. Also using the equalities $J(\cdot)=\partial\left(1 / 2\|\cdot\|^{2}\right)$ and $J_{*}(\cdot)=\partial\left(1 / 2\|\cdot\|^{2}\right)$ we obtain

$$
\begin{aligned}
\partial \varphi(x, v) & =\left(\partial_{X} \varphi(x, v), \partial_{X^{*}} \varphi(x, v)\right)+\partial h(x, v) \\
& =\left(J(x)-v_{0}, J_{*}\left(v-v_{0}\right)\right)+\partial h(x, v) .
\end{aligned}
$$

Since $X \times X^{*}$ is reflexive and $\varphi$ is lower semicontinuous and strongly convex, it attains a minimum at some $(x, v) \in X \times X^{*}$. Hence, for such $(x, v)$,

$$
0 \in\left(J(x)-v_{0}, J_{*}\left(v-v_{0}\right)\right)+\partial h(x, v) .
$$

To simplify the manipulations, define

$$
\begin{aligned}
& r=J_{*}\left(v-v_{0}\right)+x, \\
& \rho=v-v_{0}+J(x) .
\end{aligned}
$$

With this notation, the last inclusion becomes

$$
(v-\rho, x-r) \in \partial h(x, v) .
$$

Hence, by Fenchel-Young we have that

$$
h(x, v)+h^{*}(v-\rho, x-r)=\langle x, v-\rho\rangle+\langle x-r, v\rangle .
$$

Now define

$$
C:=(\langle x, v\rangle-h(x, v))+\left(\langle v-\rho, x-r\rangle-h^{*}(v-\rho, x-r)\right) .
$$

Assumption (3.1) yields $C \leq 0$. Now using (3.7), (3.6) we obtain

$$
\begin{aligned}
C & =(\langle x, v\rangle+\langle v-\rho, x-r\rangle)-(\langle x, v-\rho\rangle+\langle x-r, v\rangle) \\
& =\langle r, \rho\rangle \\
& =\langle x, J(x)\rangle+\left\langle J_{*}\left(v-v_{0}\right), v-v_{0}\right\rangle+\left\langle x, v-v_{0}\right\rangle+\left\langle J_{*}\left(v-v_{0}\right), J(x)\right\rangle .
\end{aligned}
$$


Using (2.1) and the fact that $J_{*}$ is the inverse of $J$ we have

$$
\begin{array}{ll}
\langle x, J(x)\rangle & =(1 / 2)\left(\|x\|^{2}+\|J(x)\|^{2}\right), \\
\left\langle J_{*}\left(v-v_{0}\right), v-v_{0}\right\rangle & =(1 / 2)\left(\left\|v-v_{0}\right\|^{2}+\left\|J_{*}\left(v-v_{0}\right)\right\|^{2}\right) .
\end{array}
$$

The combination of these equalities with the above expression for $C$ gives

$$
\begin{aligned}
C= & (1 / 2)\left(\|x\|^{2}+\left\|v-v_{0}\right\|^{2}+2\left\langle x, v-v_{0}\right\rangle\right) \\
& +(1 / 2)\left(\|J(x)\|^{2}+\left\|J_{*}\left(v-v_{0}\right)\right\|^{2}+2\left\langle J_{*}\left(v-v_{0}\right), J(x)\right\rangle\right) .
\end{aligned}
$$

By the above equation and Proposition 2.3. $C \geq 0$. Therefore, $C=0$. Using this fact along with (3.9) and Proposition 2.3, we conclude that $v-v_{0}=-J(x)$, that is,

$$
v+J(x)=v_{0} .
$$

On the other hand, using (3.8), (3.1) and the equality $C=0$ we conclude that $h(x, v)=\langle v, x\rangle$, which yields

$$
v \in T(x) .
$$

Therefore, $v_{0} \in(T+J)(x)$. Since $v_{0}$ is arbitrary, $T+J$ is onto.

We have thus proved that $T$ is monotone and $T+J$ is onto, hence by Proposition 2.2, $T$ is maximal monotone. It remains to prove that $h$ and $\mathcal{J}(h) \in \mathcal{H}(T)$. In order to do this, we use (3.1) and the definition of $\mathcal{H}$, for concluding that $h \in \mathcal{H}(T)$. The inclusion $\mathcal{J}(h) \in \mathcal{H}(T)$ now follows from Theorem 2.1.

\section{REFERENCES}

[1] Asplund, E.: Averaged norms, Israel Journal of Mathematics 5 (1967), 227-233. MR 36:5660

[2] Burachik, R.S. and Svaiter, B.F.: Maximal monotone operators, convex functions and a special family of enlargements, Set Valued Analysis (to appear).

[3] Moreau, J.-J.: Fonctionelles convexes, mimeographed lecture notes, Collège de France, 1967.

[4] Fitzpatrick, S.: Representing monotone operators by convex functions, Workshop/Miniconference on Functional Analysis and Optimization (Canberra, 1988) 59-65, Proc. Centre Math. Anal. Austral. Nat. Univ., 20 Austral. Nat. Univ., Canberra, 1988. MR 90i: 47054

[5] Krauss, Eckehard: A representation of maximal monotone operators by saddle functions, Rev. Roumaine Math. Pures Appl. 30 (1985), 823-837. MR 87e:47074

[6] Martinez-Legaz, J.-E. and Thera, M.: A convex representation of maximal monotone operators, Journal of Nonlinear and Convex Analysis 2 (2001), 243-247. MR 2002e:49035

[7] Rockafellar, R. T.: On the maximal monotonicity of subdifferential mappings, Pacific Journal of Mathematics 33 (1970), 209-216. MR 41:7432

[8] - On the maximality of sums of nonlinear monotone operators, Transactions of the American Mathematical Society 149 (1970), 75-88. MR 43:7984

[9] _ An extension of Fenchel's duality theorem, Duke Math. J. 33 (1966), 81-90. MR 32: 4517

Engenharia de Sistemas e Computação, COPPE-UFRJ CP 68511, Rio de Janeiro-RJ, CEP 21945-970 BRAZIL

E-mail address: regi@cos.ufrj.br

impa instituto de Matemática Pura e Aplicada, Estrada Dona Castorina 110, Rio DE JANEIRo-RJ, CEP 22460-320 BRAZIL

E-mail address: benar@impa.br 